\title{
Schlafstörungen als Folge der Therapie?
}

30-60\% der Tumorpatienten leiden unter Schlafstörungen. Möglicherweise verstärken die Therapie und die damit einhergehenden Nebenwirkungen diese noch, so das Ergebnis einer epidemiologischen Studie aus Kanada, in die 465 Patientinnen mit Brust- und 263 Patienten mit Prostatakrebs einbezogen wurden. Sie beantworteten zu sechs verschiedenen Zeitpunkten zwei Fragebögen - den ISI (Insomnia Severity Index) und einen zu somatischen Symptomen: perioperativ sowie 2, 6, 10, 14 und 18 Monate danach. Separat untersucht wurden die Effekte von Chemo-, Radio- und hormoneller Therapie.

Bei Brustkrebspatientinnen mit waren die Radiotherapie (insgesamt) und die
Chemotherapie (nach 2 Monaten) signifikant mit zunehmenden Schlafstörungen (ISI) assoziiert, nicht aber die hormonelle Therapie. Beim Prostatakarzinom ging die Androgenentzugstherapie durchweg mit erhöhten ISI-Werten einher. Dass sich für Brustkrebspatientinnen bezüglich Hormontherapie und Schlafstörungen kein signifikanter Zusammenhang zeigen ließ, geht möglicherweise auf einen Ceiling-Effekt zurück (kombinierter Einfluss aller drei Tumortherapien).

Bei Brustkrebspatientinnen wurden die Effekte von Chemo- und Radiotherapie auf den Schlaf signifikant durch eine Reihe von Symptomen vermittelt, z.B. nächtlicher Harndrang, Übelkeit oder nächtli-

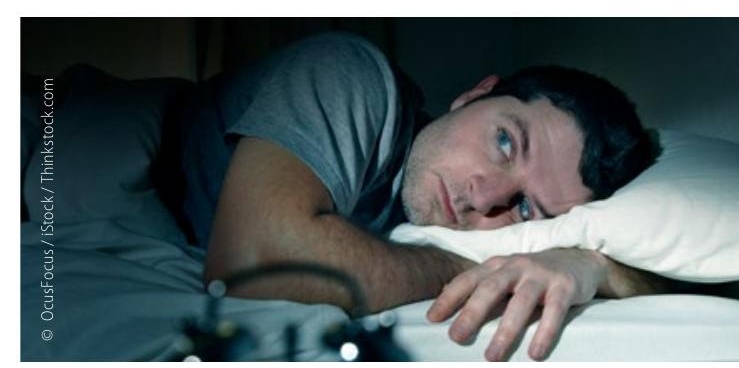

Unter einer Androgenentzugstherapie leiden Männer vermehrt unter Schlafstörungen.

che Schweißausbrüche. Letztere spielten akut und längerfristig nicht nur bei Brustkrebs-, sondern auch bei Prostatakrebspatienten eine große Rolle als Auslöser der Schlafstörungen.

Brigitte Schalhorn

Savard J et al. Cancer treatments and their side effects are associated with aggravation of insomnia: Results of a longitudinal study. Cancer. 2015;121(10):1703-11.

\section{Bisher wenig erforschtes Terrain}

\section{Thromben im Splanchnikusgebiet erste Anzeichen von Krebs?}

Venenthrombosen im Splanchnikusgebiet sind möglicherweise ein Marker für Tumoren und ein Prognosefaktor bei Patienten mit Leber- oder Pankreaskrebs, so das Ergebnisn einer dänischen Registerstudie.

Um den Zusammenhang mit Venenthrombosen im Splanchnikusgebiet (SVT) besser zu beleuchten, haben die Wissenschaftler die Befunde von 1.191 Patienten ausgewertet, bei denen Thrombosen in dieser Region des Abdomens diagnostiziert worden waren. Das Follow-up lag im Median bei 1,6 Jahren. Bei 183 dieser Pa- tienten wurde in den folgenden Monaten Krebs diagnostiziert.

An erster Stelle standen dabei Tumoren in der Leber, an zweiter Stelle im Pankreas. Bei acht Patienten wurden myeloproliferative Neoplasien diagnostiziert.

Die Wissenschaftler errechneten, dass die Wahrscheinlichkeit, innerhalb eines Vierteljahres nach der SVT-Diagnose irgendeinen Tumor zu entdecken, bei den SVT-Patienten 33-fach höher war als in der übrigen Bevölkerung (standardisierte Inzidenzrate, SIR = 33).

\section{kurz notiert}

\section{Studie zur Hochpräzisionsbestrahlung bei Lungenkrebs}

Eine Hochpräzisionsbestrahlung (stereotaktische Bestrahlung) kann bei einigen Patienten mit Lungenkrebs sehr gute Heilungsraten erzielen. Zum Einsatz kommen hierbei Bestrahlungsgeräte, die eine höhere Präzision und eine deutlich verkürzte Behandlungsdauer erlauben, z. B. der Linearbeschleuniger TrueBeam STx. In einer Studie wird nun untersucht, ob diese Form der Radiotherapie auch bei Patienten sicher und wirksam ist, deren Tumor direkt an Herz, Speiseröhre oder andere Organe anschließt. Bislang wird in diesen Fällen keine Bestrahlung vorgenommen. Ein weiteres Studienziel ist es, allgemeingültige Behandlungsstandards zu entwickeln. Denn für viele Anwendungen der Hochpräzisionsbestrahlung fehlen bisher verbindliche Leitlinien. Die Studie wird an 23 Zentren in Europa durchgeführt. Die Koordination übernimmt Prof. Dr. Ursula Nestle von der Klinik für Strahlenheilkunde des Universitätsklinikums Freiburg (ursula. nestle@uniklinik-freiburg.de).Teilnehmen können Patienten mit inoperablem nicht kleinzelligem Lungenkarzinom, die noch keine Strahlentherapie im Bereich des Brustkorbs erhalten haben.
Alle Patienten mit einem hepatozellulären Karzinom oder einem Pankreaskarzinom hatten unabhängig von der Diagnose einer SVT eine schlechte Prognose. Die Überlebensrate nach drei Monaten war jedoch in der Gruppe der Leberkrebspatienten mit SVT-Diagnose deutlich niedriger als bei Patienten ohne diese Diagnose (44 vs. $55 \%$ ). Nach einem Jahr war der Unterschied noch größer (17 vs. 30\%). Bei Patienten mit einem Pankreaskarzinom lagen die Überlebensraten nach einem Vierteljahr bei $35 \%$ versus $53 \%$. Nach einem Jahr waren die SVT als Prognosefaktor jedoch nicht mehr geeignet (15 vs. $17 \%$ ).

Die Wissenschaftler halten es für möglich, dass Thromben im Splanchnikusgebiet bei manchen Patienten die ersten Zeichen eines Tumors sind, der die Entstehung der Gerinnsel getriggert hat. Peter Leiner

Søgaard kK et al. Splanchnic venous and a prognostic factor for

cancer survival. Blood.

2015; Jun 18 (Epub

aheadoristoringer 